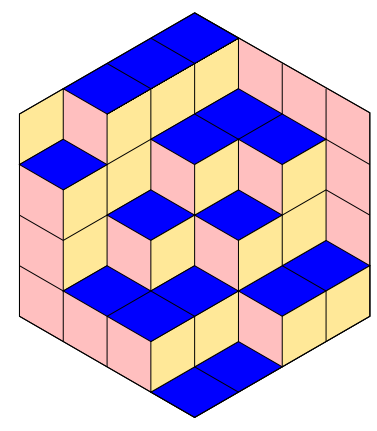

ALGEBRAIC COMBINATORICS

Greta Panova \& Piotr Śniady

Skew Howe duality and random rectangular Young tableaux

Volume 1, issue 1 (2018), p. 81-94.

<http://alco.centre-mersenne.org/item/ALCO_2018__1_1_81_0>

(C) The journal and the authors, 2018.

Some rights reserved.

(c) BY This article is licensed under the

Creative Commons ATtribution 4.0 InTERnational License.

http://creativecommons.org/licenses/by/4.0/

Access to articles published by the journal Algebraic Combinatorics on the website http://alco.centre-mersenne.org/ implies agreement with the Terms of Use (http://alco.centre-mersenne.org/legal/).

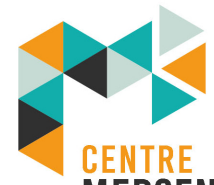

MERSENNE

Algebraic Combinatorics is member of the

Centre Mersenne for Open Scientific Publishing

www.centre-mersenne.org 


\title{
Skew Howe duality and random rectangular Young tableaux
}

\author{
Greta Panova \& Piotr Śniady
}

\begin{abstract}
We consider the decomposition into irreducible components of the external power $\bigwedge^{p}\left(\mathbb{C}^{m} \otimes \mathbb{C}^{n}\right)$ regarded as a $\mathrm{GL}_{m} \times \mathrm{GL}_{n}$-module. Skew Howe duality implies that the Young diagrams from each pair $(\lambda, \mu)$ which contributes to this decomposition turn out to be conjugate to each other, i.e. $\mu=\lambda^{\prime}$. We show that the Young diagram $\lambda$ which corresponds to a randomly selected irreducible component $\left(\lambda, \lambda^{\prime}\right)$ has the same distribution as the Young diagram which consists of the boxes with entries $\leqslant p$ of a random Young tableau of rectangular shape with $m$ rows and $n$ columns. This observation allows treatment of the asymptotic version of this decomposition in the limit as $m, n, p \rightarrow \infty$ tend to infinity.
\end{abstract}

\section{INTRODUCTION}

1.1. The Problem. In this note we address the following question. Consider

$$
\bigwedge^{p}\left(\mathbb{C}^{m} \otimes \mathbb{C}^{n}\right)=\bigoplus_{\lambda} S^{\lambda} \mathbb{C}^{m} \otimes S^{\lambda^{\prime}} \mathbb{C}^{n}
$$

as a $\mathrm{GL}_{m} \times \mathrm{GL}_{n}$-module. [...] I would like any information on the shapes of pairs of Young diagrams $\left(\lambda, \lambda^{\prime}\right)$ that give the largest contribution to the dimension asymptotically. [...]

$$
\text { Joseph M. Landsberg }[9]^{(1)}
$$

Above, $\Lambda^{p}\left(\mathbb{C}^{m} \otimes \mathbb{C}^{n}\right)$ denotes the external power of the tensor product $\mathbb{C}^{m} \otimes$ $\mathbb{C}^{n}$. Also, $S^{\lambda} \mathbb{C}^{m}$ denotes the Schur functor applied to $\mathbb{C}^{m}$ or, in other words, the irreducible representation of the general linear group $\mathrm{GL}_{m}$ with the highest weight $\lambda$. The sum in (1) runs over Young diagrams $\lambda \subseteq n^{m}$ with $p$ boxes, and such that the number of rows of $\lambda$ is bounded from above by $m$, and the number of columns of $\lambda$ is bounded from above by $n$. The decomposition (1) is nowadays referred to as skew Howe $\left(\mathrm{GL}_{m} \times \mathrm{GL}_{n}\right)$-duality, cf. [8, Theorem 4.1.1]. Even though (1) provides full information about the decomposition into irreducible components, it is not very

\footnotetext{
Manuscript received 7th August 2017, revised 21st September 2017, accepted 17th November 2017. KEYwords. Skew Howe duality, random Young diagrams, representations of general linear groups $\mathrm{GL}_{m}$, representations of finite symmetric groups.

ACKNOWLEDGEMENTS. Research of GP is partially supported by the NSF. Research of PŚ was supported by Narodowe Centrum Nauki, grant number 2014/15/B/ST1/00064.

${ }^{(1)}$ The question of Landsberg is reproduced here in a slightly redacted version. In particular, the original question considered only the special case $m=n$.
} 
convenient for answering such asymptotic questions, see the introduction to the work of Biane [3] for discussion of difficulties related to similar problems.

Despite improvements in the understanding of asymptotic problems related to the representation theory of the general linear groups $\mathrm{GL}_{m}[2,4,5]$, we do not see generic tools which would be suitable for investigation of the external power (1).

1.2. Motivations: Geometric Complexity Theory. Besides the natural interest in the question as a problem in asymptotic representation theory, this question is also relevant within Geometric Complexity Theory (GCT). The decomposition appears in the study of the complexity of matrix multiplication [10] and, in particular, in the study of the border rank of the matrix multiplication tensor as a standard measure of complexity. A lower bound for the border rank is obtained from the rank of a particular linear map, whose kernel can be decomposed as a $\mathrm{GL}(V) \times \mathrm{GL}(W)$ representation. The general approach in GCT would be to study the irreducible components for polynomials to play the role of "obstruction candidates" and, depending on the precise setup, the multiplicities would show where to find the obstructions.

1.3. The main Result. A partial answer to the question of Landsberg which we give in the current paper is based on a simple result which transforms the original problem into a question about the representation theory of the symmetric group $\mathfrak{S}_{p}$ for which more asymptotic tools are available, see Section 1.7 below.

We state our main result in two equivalent versions which are of quite distinct flavors:

- as Theorem 1.1 which is conceptually simpler and is a purely enumerative statement which relates some dimensions of the representations of the general linear groups $\mathrm{GL}_{k}$ to the dimensions of some representations of the symmetric groups $\mathfrak{S}_{p}$, and

- as Theorem 1.4 which is a probabilistic statement which relates the distribution of a random irreducible component of the external power (1) to the distribution of a random irreducible component of a certain representation of the symmetric group $\mathfrak{S}_{p}$. This second formulation is more convenient for addressing Landsberg's problem.

The proof of Theorem 1.1 is shorter, but the proof of Theorem 1.4 might be advantageous for some readers who prefer more representation-theoretic viewpoint.

1.4. The main Result: the enumerative Version. Let $m, n \geqslant 1$ be integers and let $\lambda \subseteq n^{m}$ be a Young diagram with $p$ boxes which has at most $m$ rows and at most $n$ columns. We denote by $n^{m}$ the rectangular Young diagram with $m$ rows and $n$ columns. We denote by $f^{\lambda}$ the dimension of the irreducible representation of $\mathfrak{S}_{p}$ corresponding to the Young diagram $\lambda$. Note that the skew Young diagram $n^{m} / \lambda$ is a rotation by $180^{\circ}$ of a certain Young diagram therefore it defines an irreducible representation of $\mathfrak{S}_{p}$.

TheOREM 1.1. For a Young diagram $\lambda \subseteq n^{m}$ with $p$ boxes we have the following relationship between dimensions of representations of $\mathrm{GL}_{m}, \mathrm{GL}_{n}$ and $\mathfrak{S}_{p}$ :

$$
\frac{\operatorname{dim}\left(S^{\lambda} \mathbb{C}^{m}\right) \operatorname{dim}\left(S^{\lambda^{\prime}} \mathbb{C}^{n}\right)}{\operatorname{dim} \bigwedge^{p}\left(\mathbb{C}^{m} \otimes \mathbb{C}^{n}\right)}=\frac{f^{\lambda} f^{n^{m} / \lambda}}{f^{n^{m}}}
$$

Our proof of this result (see Section 2) will be based on algebraic combinatorial manipulations with the hook-length formula and the hook-content formula. 
1.5. Bijective PRoofs? Theorem 1.1 implies the following result.

Claim 1.2. For all integers $n, m \geqslant 1$ and $0 \leqslant p \leqslant n m$ the fraction

$$
C_{n, m, p}:=\frac{f^{\lambda} f^{n^{m} / \lambda}}{\operatorname{dim}\left(S^{\lambda} \mathbb{C}^{m}\right) \operatorname{dim}\left(S^{\lambda^{\prime}} \mathbb{C}^{n}\right)}
$$

is a constant which does not depend on the choice of a Young diagram $\lambda \subseteq n^{m}$ with $p$ boxes. ${ }^{(2)}$

Conversely, Claim 1.2 implies Theorem 1.1 since

$$
C_{n, m, p} \sum_{\substack{\lambda \vdash p \\ \lambda \subseteq n^{m}}} \operatorname{dim}\left(S^{\lambda} \mathbb{C}^{m}\right) \operatorname{dim}\left(S^{\lambda^{\prime}} \mathbb{C}^{n}\right)=\sum_{\substack{\lambda \vdash p \\ \lambda \subseteq n^{m}}} f^{\lambda} f^{n^{m} / \lambda}
$$

implies

$$
C_{n, m, p} \operatorname{dim} \bigwedge^{p}\left(\mathbb{C}^{m} \otimes \mathbb{C}^{n}\right)=f^{n^{m}}
$$

(the left-hand side is an application of skew Howe duality (1)) which determines uniquely the constant

$$
C_{n, m, p}=\frac{f^{n^{m}}}{\operatorname{dim} \bigwedge^{p}\left(\mathbb{C}^{m} \otimes \mathbb{C}^{n}\right)}=\frac{f^{n^{m}}}{\left(\begin{array}{c}
n m \\
p
\end{array}\right)} .
$$

This observation opens the following challenging problem.

Problem 1.3. For a pair of Young diagrams $\lambda, \mu \subseteq n^{m}$, each with $p$ boxes, find a bijective proof of the identity

(4) $\operatorname{dim}\left(S^{\lambda} \mathbb{C}^{m}\right) \operatorname{dim}\left(S^{\lambda^{\prime}} \mathbb{C}^{n}\right) f^{\mu} f^{n^{m} / \mu}=\operatorname{dim}\left(S^{\mu} \mathbb{C}^{m}\right) \operatorname{dim}\left(S^{\mu^{\prime}} \mathbb{C}^{n}\right) f^{\lambda} f^{n^{m} / \lambda}$

which is clearly equivalent to Claim 1.2 and thus to Theorem 1.1.

Clearly, each of the factors which contribute to (4) has a natural combinatorial interpretation as the number of (semi)standard Young tableaux of some specific shape. In fact, it would be enough to find such a bijection in the special case when $\mu$ is obtained from $\lambda$ by a removal and an addition of a single box.

\subsection{The Main Result: the PRobabilistic Version.}

THEOREM 1.4. Let $m, n \geqslant 1$ and $0 \leqslant p \leqslant m n$ be integer numbers.

The random irreducible component of (1) corresponds to a pair of Young diagrams $\left(\lambda, \lambda^{\prime}\right)$, where $\lambda$ has the same distribution as the Young diagram which consists of the boxes with entries $\leqslant p$ of a uniformly random Young tableau with rectangular shape $n^{m}$ with $m$ rows and $n$ columns.

Alternatively: the random Young diagram $\lambda$ has the same distribution as a Young diagram which corresponds to a random irreducible component of the restriction $V^{n^{m}} \downarrow_{\mathfrak{S}_{p}}^{\mathfrak{S}_{m n}}$ of the irreducible representation $V^{n^{m}}$ of the symmetric group $\mathfrak{S}_{m n}$ which corresponds to the rectangular diagram $n^{m}$.

Above, when we speak about a random irreducible component of a representation we refer to the following concept.

\footnotetext{
${ }^{(2)}$ The value of the constant is clearly given by (3); we decided to state the Claim in this weaker form without the explicit value of $C_{n, m, p}$ for reasons which will become clear later.
} 


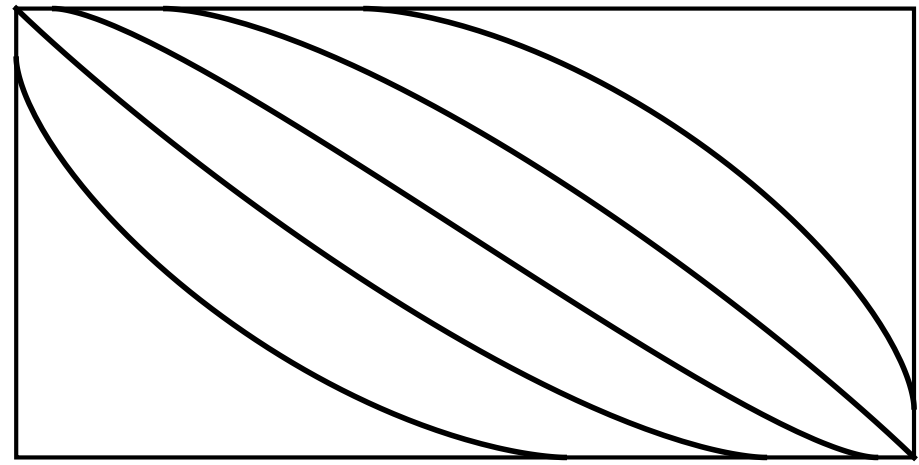

Figure 1. Asymptotic limit shapes of typical random Young diagrams which appear in Theorem 1.4, cf. [12, Figure 3]. We draw Young diagrams in the French convention. In this example the rectangle ratio is given by $\frac{m}{n}=\frac{1}{2}$. The limit curves correspond to $\frac{p}{m n} \in\left\{\frac{1}{6}, \frac{2}{6}, \frac{3}{6}, \frac{4}{6}, \frac{5}{6}\right\}$.

Definition 1.5. For a representation $V$ of a group $G$ we consider its decomposition into irreducible components

$$
V=\underset{\zeta \in \widehat{G}}{\bigoplus} m_{\zeta} V^{\zeta}
$$

where $m_{\zeta} \in\{0,1, \ldots\}$ denotes the multiplicity of $V^{\zeta}$ in $V$. This defines a probability measure $\mathbb{P}_{V}$ on the set $\widehat{G}$ of irreducible representations given by

$$
\mathbb{P}_{V}(\zeta)=\mathbb{P}_{V}^{G}(\zeta):=\frac{m_{\zeta} \operatorname{dim} V^{\zeta}}{\operatorname{dim} V}
$$

With this definition in mind, each side of the identity (2) from Theorem 1.1 can be interpreted as the probability that an appropriate random Young diagram (which appears in Theorem 1.4) has a specified shape. This provides the link between Theorem 1.1 and Theorem 1.4.

1.7. Application: BaCK to Landsberg's Problem. The problem of Landsberg is exactly a question about the statistical properties of the random Young diagram $\lambda$ which appears in Theorem 1.4. This result gives an alternative description of $\lambda$ in terms of the representation theory of the symmetric groups $\mathfrak{S}_{p}$ in which many asymptotic problems have well-known answers. Fortunately, this happens to be the case for the problem of understanding the restriction of irreducible representations which we encounter in Theorem 1.4.

In particular, the law of large numbers for the corresponding random Young diagrams has been proved in a much wider generality by Biane [3, Theorem 1.5.1] using the language of free cumulants of Young diagrams. The asymptotic Gaussianity of their fluctuations around the limit shape has been proved by the second-named author [13, Example 7 combined with Theorem 8] using the same language.

In the specific case of the restriction $V^{n^{m}} \downarrow_{\mathfrak{S}_{p}}^{\mathfrak{S}_{m n}}$ which is in the focus of the current paper, the above-mentioned generic tools $[3,13]$ can be applied in the scaling when $m, n, p \rightarrow \infty$ tend to infinity in such a way that the rectangle ratio $\frac{m}{n}$ converges to a strictly positive limit and the fraction $\frac{p}{m n}$ converges to some limit. Pittel and Romik [12] have worked out this specific example and, among other results, found explicit asymptotic limit shapes of typical Young diagrams which contribute to such representations, see Figure 1. In the light of Theorem 1.4, the above references provide 
a partial answer to the question of Landsberg. For more on this topic see the work of Sevak Mkrtchyan [11].

1.8. Hypothetical extensions of Theorem 1.4. The formulation of Theorem 1.4 might suggest that it is a special case of a more general result. We state it concretely as the following problem.

Problem 1.6. Find a natural quantum random walk (in the spirit of Biane [1]) on the set of irreducible representations (of some group? of some algebra?) with the property that the probability distribution on the set of paths of this random walk can be identified (via some hypothetical analogue of Theorem 1.4) with the uniform distribution on the set of standard Young tableaux of rectangular shape $n^{m}$.

\section{Proof of Theorem 1.1}

First, we give an enumerative proof of Theorem 1.1 using the classical dimension formulas: the hook-length formula for $f^{\nu}$

$$
f^{\nu}=\frac{|\nu| !}{\prod_{\square \in \nu} h_{\square}}=: \frac{|\nu| !}{H_{\nu}},
$$

where $H_{\nu}$ denotes the product of hook lengths in $\nu$, and the hook-content formula for the dimensions of representations of $\mathrm{GL}_{k}$ :

$$
\operatorname{dim} S^{\nu} \mathbb{C}^{k}=s_{\nu}\left(1^{k}\right)=\frac{\prod_{\square \in \nu}(k+c(\square))}{\prod_{\square \in \nu} h_{\square}},
$$

where $h_{\square}$ is the hook-length of a box $\square$ in the diagram of $\nu$, and the content $c(\square)=$ $j-i$, if $\square=(i, j)$ is at row $i$ and column $j$ of the diagram. Here $s_{\nu}\left(x_{1}, \ldots, x_{k}\right)$ is the corresponding Schur function.

\section{Claim 2.1. We have that}

$$
s_{\nu}\left(1^{n}\right)=s_{m^{n} / \nu}\left(1^{n}\right)=s_{\bar{\nu}}\left(1^{n}\right)
$$

for any $\nu \subseteq m^{n}$, where $\bar{\nu}=\left(m-\nu_{n}, m-\nu_{n-1}, \ldots, m-\nu_{1}\right)$ is the complementary partition.

Proof. Representation-theoretic proof: the left-hand side of (6) is equal to the dimension of the representation $S^{\nu} \mathbb{C}^{n}$ of $\mathrm{GL}_{n}$ which corresponds to the Young diagram $\nu$ while the right-hand side is equal to the dimension of the tensor product of the one-dimensional representation $\mathrm{GL}_{n} \ni g \mapsto(\operatorname{det} g)^{m}$ with the representation contragradient to $S^{\nu} \mathbb{C}^{n}$. Their dimensions are clearly equal.

Combinatorial (bijective) proof: SSYTs with entries $1, \ldots, n$ of shape $\nu$ correspond to SSYTs with entries $1, \ldots, n$ and shape $\bar{\nu}$ via the following bijection. Consider an SSYT $T$ of shape $\nu$ as sitting inside the $m^{n}$ rectangle. In a given column $j$ of $m^{n}$, let $a_{1}<\cdots<a_{\nu_{j}^{\prime}}$ be the entries of $T$ in this column. Let $\left\{b_{1}<b_{2}<\cdots<b_{n-\nu_{j}^{\prime}}\right\}:=$ $\{1, \ldots, n\} \backslash\left\{a_{1}, \ldots, a_{\nu_{j}^{\prime}}\right\}$ be the remaining numbers in $\{1, \ldots, n\}$. Write them in increasing order top to bottom in the column $j$ in $m^{n}$ above $T$ as in Figure 2a; note that we use the French convention for drawing Young diagrams. Rotating the resulting tableau above $T$ by $180^{\circ}$ we obtain a SSYT of shape $\bar{\nu}$ with entries in $\{1, \ldots, n\}$ as in Figure $2 \mathrm{~b}$ (the row inequalities are easily seen to be satisfied).

We continue the proof of Theorem 1.1. Using the claim for $\nu:=\lambda^{\prime}$ we have that

$$
\operatorname{dim} S^{\lambda^{\prime}} \mathbb{C}^{n}=s_{\lambda^{\prime}}\left(1^{n}\right)=s_{\overline{\lambda^{\prime}}}\left(1^{n}\right)=\frac{\prod_{\square \in \overline{\lambda^{\prime}}}(n+c(\square))}{H_{\overline{\lambda^{\prime}}}}=\frac{\prod_{\square \in \overline{\lambda^{\prime}}}(n+c(\square))}{H_{\bar{\lambda}}}
$$


The last equality follows from the observation that $\overline{\lambda^{\prime}}=(\bar{\lambda})^{\prime}$, where the second complement is taken in the $n^{m}$ rectangle and so $H_{\overline{\lambda^{\prime}}}=H_{\bar{\lambda}}$.

Consider the partitions $\lambda$ and $\overline{\lambda^{\prime}}$ as sitting inside $n^{m}$. More specifically, any box

$$
\square^{\prime}=\left(i^{\prime}, j^{\prime}\right) \in \overline{\lambda^{\prime}}
$$

\begin{tabular}{|l|l|l|l|l|l|l|l|}
\hline 4 & 4 & 2 & 1 & 1 & 1 & 1 & 1 \\
\hline 3 & 3 & 4 & 4 & 4 & 2 & 2 & 2 \\
\hline 2 & 2 & 3 & 3 & 3 & 4 & 4 & 3 \\
\hline 1 & 1 & 1 & 2 & 2 & 3 & 3 & 4 \\
\hline
\end{tabular}

(a)

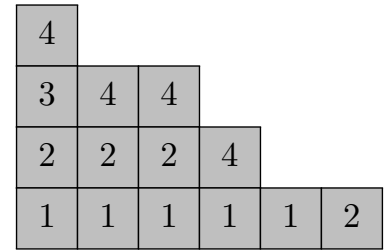

(b)

Figure 2. An example of the bijection from the proof of Claim 2.1 with $m=8, n=4$, and $\nu=(7,5,4,2)$. (a) The original tableau is in white, and the (not rotated yet) complementary tableau of skew shape $m^{n} / \nu$ is in gray. (b) The complementary tableau of shape $\bar{\nu}=$ $(6,4,3,1)$.

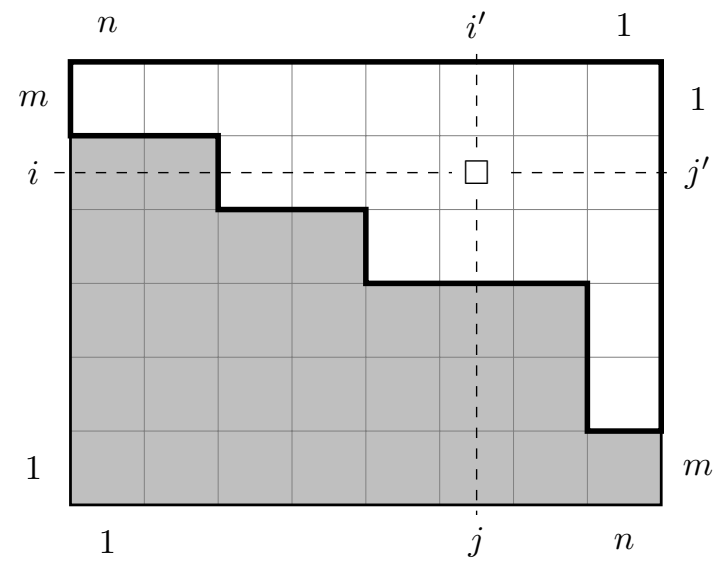

(a)

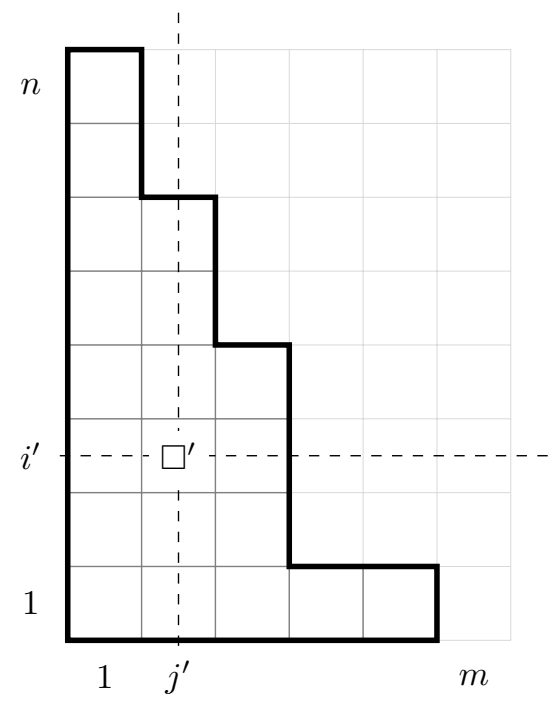

(b)

Figure 3. The relationship between contents within $\lambda, \overline{\lambda^{\prime}}$ and the rectangle $n^{m}$. (a) In this example $m=6, n=8, \lambda=$ $(8,7,7,4,2)$ is drawn with gray background. (b) The partition $\overline{\lambda^{\prime}}=$ $(5,3,3,3,2,2,1,1)$ is drawn with white background. Figure (a) shows how $\lambda$ and $\overline{\lambda^{\prime}}$ are combined together within the rectangle $n^{m}$ and how their respective coordinate systems are related. The content of $\square^{\prime}=\left(i^{\prime}, j^{\prime}\right)=(3,2) \in \overline{\lambda^{\prime}}$ is $c\left(\square^{\prime}\right)=2-3=-1$, the same box in $n^{m}$ is $\square=(i, j)=(5,6)$ with $c(\square)=1$ and $n+c\left(\square^{\prime}\right)=7=m+c(\square)$. 
corresponds to the box

$$
\square=(i, j)=\left(m+1-j^{\prime}, n+1-i^{\prime}\right) \in n^{m},
$$

see Figure 3, and so the content $c\left(\square^{\prime}\right)=c_{\bar{\lambda}^{\prime}}\left(\square^{\prime}\right)$ of $\square$ regarded as a box of $\overline{\lambda^{\prime}}$ fulfills

$$
n+c_{\bar{\lambda}^{\prime}}\left(\square^{\prime}\right)=n+j^{\prime}-i^{\prime}=n+(m+1-i)-(n+1-j)=m+c_{n^{m}}(\square),
$$

where the content $c(\square)=c_{n^{m}}(\square)$ is taken with respect to the $n^{m}$ partition.

Since $s_{n^{m}}\left(1^{m}\right)=1$ (there is only one SSYT of shape $n^{m}$ and entries $1, \ldots, m$ since each column is forced to be $1, \ldots, m)$, it follows from the hook-content formula (5) that

$$
\prod_{\square \in n^{m}}(m+c(\square))=H_{n^{m}}
$$

Thus by (7), then by combining the diagrams $\lambda$ and $\overline{\lambda^{\prime}}$ into the $n^{m}$ rectangle, and by (8)

$$
\begin{aligned}
\operatorname{dim} S^{\lambda} \mathbb{C}^{m} \operatorname{dim} S^{\lambda^{\prime}} \mathbb{C}^{n} & =s_{\lambda}\left(1^{m}\right) s_{\lambda^{\prime}}\left(1^{n}\right) \\
& =\frac{\prod_{\square \in \lambda}(m+c(\square))}{H_{\lambda}} \frac{\prod_{\square^{\prime} \in n^{m} / \lambda}\left(m+c\left(\square^{\prime}\right)\right)}{H_{\bar{\lambda}}} \\
& =\frac{\prod_{\square \in n^{m}}(m+c(\square))}{H_{\lambda} H_{\bar{\lambda}}}=\frac{H_{n^{m}}}{H_{\lambda} H_{\bar{\lambda}}} \\
& =\frac{(m n) ! / f^{n^{m}}}{\left(p ! / f^{\lambda}\right)\left((m n-p) ! / f^{n^{m} / \lambda}\right)}=\left(\begin{array}{c}
m n \\
p
\end{array}\right) \frac{f^{\lambda} f^{n^{m} / \lambda}}{f^{n^{m}}} .
\end{aligned}
$$

This concludes the proof of Theorem 1.1.

REMARK 2.2. Relationships between $H_{\lambda}, H_{\bar{\lambda}}$ and Schur function evaluations have also been derived by Stanley [14] who used them further in computations of the normalized symmetric group character corresponding to rectangular partitions.

\section{Proof of Theorem 1.4}

3.1. SketCH OF THE PROOF. We start by presenting a one-paragraph summary of the proof. Schur-Weyl duality suggests exploring the link between the structure of the external power (1) viewed as a representation of some general linear group $\mathrm{GL}_{m}$ and the structure of the same space (1), this time viewed as a representation of the symmetric group $\mathfrak{S}_{p}$. Regretfully, the external power (1) is not a representation of $\mathfrak{S}_{p}$. This approach can be rescued if, instead, we view the external power as a module over the center $Z \mathbb{C}\left[\mathfrak{S}_{p}\right]$ of the symmetric group algebra. The character theory of the symmetric group $\mathfrak{S}_{p}$ can be easily adapted to the setting of $Z \mathbb{C}\left[\mathfrak{S}_{p}\right]$. We will show that the characters of $\bigwedge^{p}\left(\mathbb{C}^{m} \otimes \mathbb{C}^{n}\right)$ (for fixed values of $m$ and $n$ and for $p$ varying over $\{0,1, \ldots, m n\})$ are closely related to each other; in this way it is enough to identify such a character for $p=m n$. Yet another application of Schur-Weyl duality shows that this particular character is irreducible and corresponds to the rectangular Young diagram $n^{m}$.

In the remaining part of this section we will present the details of the above sketch. For clarity the proof is split into a number of propositions. 
3.2. Normalized Characters. In this paper whenever we refer to a trace of a matrix $A=\left(A_{i j}\right)_{1 \leqslant i, j \leqslant d} \in M_{d}(\mathbb{C})$ we mean the normalized trace

$$
\operatorname{tr}_{d} A:=\frac{1}{d} \sum_{1 \leqslant i \leqslant d} A_{i i}
$$

as opposed to the non-normalized trace

$$
\operatorname{Tr} A:=\sum_{1 \leqslant i \leqslant d} A_{i i} .
$$

For an operator $A \in$ End $V$ we denote by $\operatorname{tr}_{V} A$ its normalized trace, defined analogously.

Also, by the character of a group representation $\rho: G \rightarrow$ End $V$ we mean the normalized character $\chi_{V}: G \rightarrow \mathbb{C}$ given by

$$
\chi_{V}(g):=\operatorname{tr}_{V} \rho_{g}
$$

which is defined in terms of the normalized $\operatorname{trace}_{V} \operatorname{tr}_{V}$.

3.3. Modules over the Center $Z \mathbb{C}[G]$ of the Group ALGeBra. In the following we will consider the following setup. We assume that $G$ is a finite group and $W$ is a $G$-module. We also assume that $\Pi: W \rightarrow W$ is an idempotent $\Pi^{2}=\Pi$ with the property that $\Pi$ commutes with the action of the center $Z \mathbb{C}[G]$ of the group algebra. We denote by $V:=\Pi W$ the image of $\Pi$. The space $V$ is invariant under the action of $Z \mathbb{C}[G]$; in other words $V$ can be regarded as a $Z \mathbb{C}[G]$-module. by

We define the character of the $Z \mathbb{C}[G]$-module $V$ as a function $\chi_{V}: G \rightarrow \mathbb{C}$ given

$$
\chi_{V}(g):=\frac{1}{\operatorname{dim} V} \operatorname{Tr}\left[\Pi \rho_{g}\right],
$$

where $\rho_{g}: W \rightarrow W$ denotes the action of $g \in G$ on $W$.

Our goal in this proof is to understand $V$ as a $Z \mathbb{C}[G]$-module and to identify the corresponding character.

3.4. The KeY EXAmple. The key example we should keep in mind is the tensor product

$$
W=W_{p}:=\left(\mathbb{C}^{m}\right)^{\otimes p} \otimes\left(\mathbb{C}^{n}\right)^{\otimes p}
$$

which carries a natural structure of $G$-module, where

$$
G=G_{p}:=\mathfrak{S}_{p} \times \mathfrak{S}_{p}
$$

is the Cartesian product of the symmetric groups which acts on $W$ by permuting the factors in the tensor product.

By rearranging the order of the factors we see that

$$
W \cong\left(\mathbb{C}^{m} \otimes \mathbb{C}^{n}\right)^{\otimes p}
$$

is a tensor power which carries another structure, this time of a $\mathfrak{S}_{p}$-module. This action of $\mathfrak{S}_{p}$ is related to the action from (11) via the diagonal inclusion of groups given by

$$
\mathfrak{S}_{p} \ni g \mapsto(g, g) \in \mathfrak{S}_{p} \times \mathfrak{S}_{p} .
$$

We consider the projection $\Pi$ which is given by the action on (12) of the central projection

$$
\Pi=\Pi_{p}:=\frac{1}{p !} \sum_{g \in \mathfrak{S}_{p}}(-1)^{g} g \in Z \mathbb{C}\left[\mathfrak{S}_{p}\right]
$$


which under the inclusion (13) becomes the action on (11) of the element

$$
\Pi=\frac{1}{p !} \sum_{g \in \mathfrak{S}_{p}}(-1)^{g}(g, g) \in \mathbb{C}\left[\mathfrak{S}_{p} \times \mathfrak{S}_{p}\right]
$$

which is not central.

The image $V=\Pi W$ of this projection is the external power

$$
V=V_{p}:=\bigwedge^{p}\left(\mathbb{C}^{m} \otimes \mathbb{C}^{n}\right)
$$

which is in the focus of the current paper.

3.5. Modules over the Center $Z \mathbb{C}[G]$, Revisited. Consider now a more general situation than in Section 3.3 in which $V$ is an arbitrary $Z \mathbb{C}[G]$-module, without any additional structure.

3.5.1. Characters of $Z \mathbb{C}[G]$-modules. We can define the character $\chi_{V}: G \rightarrow \mathbb{C}$ of $V$ by the formula

$$
\chi_{V}(g):=\operatorname{tr}_{V} \rho\left[\frac{1}{|G|} \sum_{h \in G} h g h^{-1}\right] .
$$

Note that in the specific setup considered in Section 3.3 the formulas (10) and (16) define the same function. Also, in the specific setup in which the structure of a $Z \mathbb{C}[G]$ module on $V$ arises from the structure of a $G$-module, the usual character of the group $G$ given by (9) coincides with the character from (16).

3.5.2. Irreducible $Z \mathbb{C}[G]$-modules versus irreducible $G$-modules. The algebra $Z \mathbb{C}[G]$ is commutative, hence each irreducible $Z \mathbb{C}[G]$-module is one-dimensional, contrary to irreducible $G$-modules. Nevertheless, irreducible $Z \mathbb{C}[G]$-modules and irreducible $G$-modules are intimately related; we shall review this relationship in the following. In particular we shall decribe the canonical bijection between (equivalence classes of) irreducible $Z \mathbb{C}[G]$-modules and (equivalence classes of) irreducible $G$-modules.

By Schur's lemma, each central element from $Z \mathbb{C}[G]$ acts on an irreducible $G$ module $V$ as a scalar multiple of identity. In particular, it follows that $V$ - viewed this time as a $Z \mathbb{C}[G]$-module - is a sum of $\operatorname{dim} V$ copies of some irreducible onedimensional $Z \mathbb{C}[G]$-module which we denote by $V^{Z \mathbb{C}[G]}$. We claim that the map $V \mapsto V^{Z \mathbb{C}[G]}$ provides a bijection between (equivalence classes of) irreducible $G$ modules and (equivalence classes of) irreducible $Z \mathbb{C}[G]$-modules. Indeed, the characters of the corresponding modules are equal: $\chi_{V}=\chi_{V Z \mathbb{C}[G]}$ thus if two irreducible $G$ modules $V_{1}, V_{2}$ are not equivalent then the corresponding irreducible $Z \mathbb{C}[G]$-modules $V_{1}^{Z \mathbb{C}[G]}$ and $V_{2}^{Z \mathbb{C}[G]}$ are also not equivalent; it follows that the map $V \mapsto V^{Z \mathbb{C}[G]}$ is injective. The surjectivity follows from comparison of the cardinalities: the number of (equivalence classes of) irreducible modules over the commutative algebra $Z \mathbb{C}[G]$ is equal to the dimension of the latter algebra which happens to be the number of conjugacy classes of $G$; the latter number is also the number of (equivalence classes of) irreducible representations of $G$.

3.5.3. Probability distribution associated to a $Z \mathbb{C}[G]$-module. Thanks to the correspondence between irreducible $Z \mathbb{C}[G]$-modules and irreducible $G$-modules which was discussed in Section 3.5.2, any $Z \mathbb{C}[G]$-module $V$ defines (analogously as in Definition 1.5) a probability measure $\mathbb{P}_{V}^{Z \mathbb{C}[G]}$ on the set of irreducible representations of $G$. Note that in the specific situation when the structure of a $Z \mathbb{C}[G]$-module on $V$ arises from the structure of a $G$-module, the corresponding measures are equal: $\mathbb{P}_{V}^{G}=\mathbb{P}_{V}^{Z \mathbb{C}[G]}$, no matter if we regard $V$ as a $G$-module or as a $Z \mathbb{C}[G]$-module. 
These probability measures are directly related to the character of the corresponding module:

$$
\mathbb{P}_{V}(\zeta)=\chi_{V}\left(p_{\zeta}\right)=\sum_{g \in G} \chi_{V}(g) p_{\zeta}(g),
$$

where $p_{\zeta} \in Z \mathbb{C}[G]$ is the minimal central projection which corresponds to the irreducible representation $\zeta \in \widehat{G}$.

\subsection{SChuR-Weyl DUAlity. $\mathrm{GL}_{m}$ Versus $\mathfrak{S}_{p}$.}

PROPOSITION 3.1. The probability distributions of the following two pairs of random Young diagrams are equal:

- the pair $(\lambda, \mu)$ of random Young diagrams which correspond to a random irreducible component of $\bigwedge^{p}\left(\mathbb{C}^{m} \otimes \mathbb{C}^{n}\right)$ regarded as $\mathrm{GL}_{m} \times \mathrm{GL}_{n}$-module, and

- the pair $(\lambda, \mu)$ of random Young diagrams which correspond to a random irreducible component of $\bigwedge^{p}\left(\mathbb{C}^{m} \otimes \mathbb{C}^{n}\right)$ regarded as $Z \mathbb{C}\left[\mathfrak{S}_{p}\right] \times Z \mathbb{C}\left[\mathfrak{S}_{p}\right]$-module.

In the following we shall present the missing details of notation and the proof of this proposition.

The tensor power

$$
\left(\mathbb{C}^{m} \otimes \mathbb{C}^{n}\right)^{\otimes p} \cong\left(\mathbb{C}^{m}\right)^{\otimes p} \otimes\left(\mathbb{C}^{n}\right)^{\otimes p}
$$

carries a natural structure of a $\left(\mathrm{GL}_{m} \times \mathfrak{S}_{p}\right) \times\left(\mathrm{GL}_{n} \times \mathfrak{S}_{p}\right)$-module and, more generally, a structure of a $\left(\mathrm{GL}_{m} \times \mathbb{C}\left[\mathfrak{S}_{p}\right]\right) \times\left(\mathrm{GL}_{n} \times \mathbb{C}\left[\mathfrak{S}_{p}\right]\right)$-module: each of the two general linear groups $\mathrm{GL}_{m}$ and $\mathrm{GL}_{n}$ acts on all corresponding factors $\mathbb{C}^{m}$ (respectively, $\mathbb{C}^{n}$ ), while each of the two symmetric groups $\mathfrak{S}_{p}$ acts by permuting the factors in the tensor product. Regretfully, its subspace

$$
\bigwedge^{p}\left(\mathbb{C}^{m} \otimes \mathbb{C}^{n}\right)
$$

which is in the focus in the current paper is not invariant under the action of the symmetric groups $\mathfrak{S}_{p}$ which are factors in

$$
\left(\mathrm{GL}_{m} \times \mathfrak{S}_{p}\right) \times\left(\mathrm{GL}_{n} \times \mathfrak{S}_{p}\right)
$$

and under the action of the corresponding symmetric group algebras $\mathbb{C}\left[\mathfrak{S}_{p}\right]$.

On the bright side, the space (19) is invariant under the action of each of the centers $Z \mathbb{C}\left[\mathfrak{S}_{p}\right]$ of the two symmetric group algebra as well as their product $Z \mathbb{C}\left[\mathfrak{S}_{p}\right] \times Z \mathbb{C}\left[\mathfrak{S}_{p}\right]$; indeed, this is a special case of the situation considered in Section 3.3 in the setting presented in Section 3.4.

Thus both (18) and (19) carry a structure of a $\left(\mathrm{GL}_{m} \times Z \mathbb{C}\left[\mathfrak{S}_{p}\right]\right) \times\left(\mathrm{GL}_{n} \times Z \mathbb{C}\left[\mathfrak{S}_{p}\right]\right)$ module. Our proof of Theorem 1.4 will be based on exploration of this module structure.

Proof of Proposition 3.1. Let us decompose the $\left(\mathrm{GL}_{m} \times Z \mathbb{C}\left[\mathfrak{S}_{p}\right]\right) \times\left(\mathrm{GL}_{n} \times Z \mathbb{C}\left[\mathfrak{S}_{p}\right]\right)$ module (19) into irreducible components:

$$
\bigwedge^{p}\left(\mathbb{C}^{m} \otimes \mathbb{C}^{n}\right)=\bigoplus_{\lambda, \nu, \mu, \pi} m_{\lambda, \nu, \mu, \pi} S^{\lambda}\left(\mathbb{C}^{m}\right) \otimes V^{\nu} \otimes S^{\mu}\left(\mathbb{C}^{n}\right) \otimes V^{\pi},
$$

where $m_{\lambda, \nu, \mu, \pi} \in\{0,1, \ldots\}$ denotes the multiplicity, $V^{\nu}$ denotes the irreducible representation of the symmetric group $\mathfrak{S}_{p}$ which corresponds to the Young diagram $\nu$ and the sum runs over Young diagrams $\lambda, \nu, \mu, \pi$.

Schur-Weyl duality implies that a decomposition (analogous to (20)) of the tensor power (18) into irreducible components (no matter whether we regard (18) as a $\left(\mathrm{GL}_{m} \times \mathfrak{S}_{p}\right) \times\left(\mathrm{GL}_{n} \times \mathfrak{S}_{p}\right)$-module or as a $\left(\mathrm{GL}_{m} \times Z \mathbb{C}\left[\mathfrak{S}_{p}\right]\right) \times\left(\mathrm{GL}_{n} \times Z \mathbb{C}\left[\mathfrak{S}_{p}\right]\right)$-module $)$ 
involves only summands for which $\nu=\lambda$ and $\pi=\mu$. It follows that the same is true for its $\left(\mathrm{GL}_{m} \times Z \mathbb{C}\left[\mathfrak{S}_{p}\right]\right) \times\left(\mathrm{GL}_{n} \times Z \mathbb{C}\left[\mathfrak{S}_{p}\right]\right)$-submodule (19), thus the decomposition (20) takes a simpler form

$$
\bigwedge^{p}\left(\mathbb{C}^{m} \otimes \mathbb{C}^{n}\right)=\bigoplus_{\lambda, \mu} m_{\lambda, \mu} S^{\lambda}\left(\mathbb{C}^{m}\right) \otimes V^{\lambda} \otimes S^{\mu}\left(\mathbb{C}^{n}\right) \otimes V^{\mu}
$$

for some multiplicities $m_{\lambda, \mu}:=m_{\lambda, \lambda, \mu, \mu}$.

The linear span of all irreducible components of (21) which correspond to a given pair of Young diagrams $(\lambda, \mu)$ remains the same, no matter if we regard (21) as a $\mathrm{GL}_{m} \times \mathrm{GL}_{n}$-module or as a $Z \mathbb{C}\left[\mathfrak{S}_{p}\right] \times Z \mathbb{C}\left[\mathfrak{S}_{p}\right]$ module. It follows that the corresponding probability distributions are equal. This concludes the proof of Proposition 3.1.

Proposition 3.1 shows that in order to prove Theorem 1.4 it is enough to understand the structure of $\bigwedge^{p}\left(\mathbb{C}^{m} \otimes \mathbb{C}^{n}\right)$ as a $Z \mathbb{C}\left[\mathfrak{S}_{p}\right] \times Z \mathbb{C}\left[\mathfrak{S}_{p}\right]$-module. We shall do it in the following.

3.7. Characters of the external Power do not DePend on the EXPonent. We come back to the specific setup from Section 3.4. Assume that $0 \leqslant p<p^{\prime} \leqslant m n$. There is a natural inclusion $G_{p}=\mathfrak{S}_{p} \times \mathfrak{S}_{p} \subseteq \mathfrak{S}_{p^{\prime}} \times \mathfrak{S}_{p^{\prime}}=G_{p^{\prime}}$ of the corresponding groups which allows us to compare the characters of the external powers

$$
V_{p}:=\bigwedge^{p}\left(\mathbb{C}^{m} \otimes \mathbb{C}^{n}\right)
$$

for various values of the exponent $p$.

Lemma 3.2. For $0 \leqslant p<p^{\prime} \leqslant m n$ the character $\chi_{V_{p}}: G_{p} \rightarrow \mathbb{C}$ is equal to the restriction of the character $\chi_{V_{p^{\prime}}}: G_{p^{\prime}} \rightarrow \mathbb{C}$.

Proof. It is enough to prove this result in the case when $p^{\prime}=p+1$.

Let $g=\left(\pi_{1}, \pi_{2}\right) \in G_{p}=\mathfrak{S}_{p} \times \mathfrak{S}_{p}$. We denote by $\pi_{1}^{\prime}, \pi_{2}^{\prime} \in \mathfrak{S}_{p+1}$ the corresponding permutations from the larger symmetric group; in this way $g$ corresponds to $g^{\prime}=$ $\left(\pi_{1}^{\prime}, \pi_{2}^{\prime}\right) \in G_{p+1}$.

We consider the decomposition

$$
W_{p+1}=\left(\mathbb{C}^{m} \otimes \mathbb{C}^{n}\right)^{\otimes(p+1)}=W_{p} \otimes\left(\mathbb{C}^{m} \otimes \mathbb{C}^{n}\right) .
$$

With respect to this decomposition, the action of $\rho_{g^{\prime}}$ on $W_{p+1}$ coincides with the action of $\rho_{g} \otimes 1$ :

$$
\rho_{g^{\prime}}=\rho_{g} \otimes 1
$$

The projection $\Pi_{p+1}$ viewed as in (14) as an element of $\mathbb{C}\left[\mathfrak{S}_{p+1}\right]$ can be written as the product

$$
\Pi_{p+1}=\frac{1}{p+1}\left[1-X_{p+1}\right] \Pi_{p}
$$

where

$$
X_{p+1}=(1, p+1)+\cdots+(p, p+1) \in \mathbb{C}\left[\mathfrak{S}_{p+1}\right]
$$

is a Jucys-Murphy element; above $(i, j) \in \mathfrak{S}_{p+1}$ denotes the transposition which interchanges $i$ with $j$. We view now (24) as an operator acting on (22); with this perspective

$$
\Pi_{p+1}=\frac{1}{p+1}\left[1-X_{p+1}\right]\left(\Pi_{p} \otimes 1\right)
$$

is an element of $\operatorname{End}\left(W_{p}\right) \otimes \operatorname{End}\left(\mathbb{C}^{m} \otimes \mathbb{C}^{n}\right)$. 
A direct calculation on the elementary tensors shows that application of the (nonnormalized) trace $\operatorname{Tr}: \operatorname{End}\left(\mathbb{C}^{m} \otimes \mathbb{C}^{n}\right) \rightarrow \mathbb{C}$ to the second factor in (25) yields a multiple of identity:

$$
(1 \otimes \operatorname{Tr}) \frac{1}{p+1}\left[1-X_{p+1}\right]=\frac{m n-p}{p+1} .
$$

By combining this idea with (23), (25) it follows that

$$
\chi_{V_{p+1}}\left(g^{\prime}\right)=\frac{1}{\left(\begin{array}{c}
m n \\
p+1
\end{array}\right)} \operatorname{Tr}\left[\Pi_{p+1} \rho_{g^{\prime}}\right]=\frac{1}{\left(\begin{array}{c}
m n \\
p
\end{array}\right)} \operatorname{Tr}\left[\Pi_{p} \rho_{g}\right]=\chi_{V_{p}}(g)
$$

which concludes the proof.

3.8. The CHARACTER OF $\bigwedge^{p}\left(\mathbb{C}^{m} \otimes \mathbb{C}^{n}\right)$. Until now we considered the symmetric group $\mathfrak{S}_{p}$ as the diagonal subgroup of $\mathfrak{S}_{p} \times \mathfrak{S}_{p}$ via (13). In the following we take a different perspective and we shall view the symmetric group

$$
\mathfrak{S}_{p} \cong \mathfrak{S}_{p} \times\{\mathrm{id}\} \subseteq \mathfrak{S}_{p} \times \mathfrak{S}_{p}=G_{p}
$$

as the first factor in the Cartesian product.

In this way the space $W_{p}=\left(\mathbb{C}^{m}\right)^{\otimes p} \otimes\left(\mathbb{C}^{n}\right)^{\otimes p}$ has a structure of a $\mathfrak{S}_{p}$-module and $V_{p}=\bigwedge^{p}\left(\mathbb{C}^{m} \otimes \mathbb{C}^{n}\right)$ a structure of a $Z \mathbb{C}\left[\mathfrak{S}_{p}\right]$-module.

Corollary 3.3. The character $\chi_{V_{p}}: \mathfrak{S}_{p} \rightarrow \mathbb{C}$ of $Z \mathbb{C}\left[\mathfrak{S}_{p}\right]$-module $V_{p}=\bigwedge^{p}\left(\mathbb{C}^{m} \otimes \mathbb{C}^{n}\right)$ is equal to the restriction of the irreducible character $\chi_{m^{n}}: \mathfrak{S}_{m n} \rightarrow \mathbb{C}$ of the symmetric group $\mathfrak{S}_{m n}$ which corresponds to the rectangular Young diagram $\mathrm{m}^{n}$.

Proof. In the light of Lemma 3.2 it is enough to prove this result for the maximal possible value $p=m n$ and to show the equality

$$
\chi_{V_{m n}}=\chi_{m^{n}} .
$$

We shall do it in the following.

For $p=m n$ the external power $V_{m n}$ is a one-dimensional representation of $\mathrm{GL}_{m} \times \mathrm{GL}_{n}$ which corresponds to a pair of Young diagrams

$$
\left(m^{n}, n^{m}\right) \text {. }
$$

The corresponding random pair of Young diagrams (associated via Definition 1.5) is deterministic, equal to (27). Proposition 3.1 shows that if we view $V_{m n}$ as a $Z \mathbb{C}\left[\mathfrak{S}_{m n}\right] \times Z \mathbb{C}\left[\mathfrak{S}_{m n}\right]$-module, the corresponding pair of random Young diagrams is also deterministic, equal to (27). If we regard $V_{m n}$ as $Z \mathbb{C}\left[\mathfrak{S}_{m n}\right]$-module, this implies that its character is equal to the character of the irreducible representation $V^{m^{n}}$. This concludes the proof.

3.9. Pair of Random Young Diagrams $(\lambda, \mu)$. One of the claims in Theorem 1.4 is that the random Young diagrams $\lambda, \mu$ that correspond to the random irreducible component of (1) are related to each other by the equality $\mu=\lambda^{\prime}$. This result would follow from the decomposition (1). In order to be self-contained we present below a short proof based on the original ideas of Howe [8, Section 4.1.2].

Application of Schur-Weyl duality (Proposition 3.1) changes this into a problem of understanding the structure of the external power $V_{p}$ viewed as a $Z \mathbb{C}\left[\mathfrak{S}_{p}\right] \times Z \mathbb{C}\left[\mathfrak{S}_{p}\right]$ module. For any permutation $g_{2} \in \mathfrak{S}_{p}$ the action of $\left(g_{2}^{-1}, g_{2}^{-1}\right) \in \mathfrak{S}_{p} \times \mathfrak{S}_{p}$ on the external power $V_{p}$ coincides with the multiple of identity $(-1)^{g_{2}}$. It follows that for any $g_{1}, g_{2} \in \mathfrak{S}_{p}$

$$
\chi_{V_{p}}\left(g_{1}, g_{2}\right)=(-1)^{g_{2}} \chi_{V_{p}}\left(g_{1} g_{2}^{-1}, \mathrm{id}\right)
$$


By linearity, it follows that for the minimal central projections $p_{\lambda}, p_{\mu} \in Z \mathbb{C}\left[\mathfrak{S}_{p}\right]$ which correspond to the Young diagrams $\lambda, \mu$ we have

$$
\begin{aligned}
\chi_{V_{p}}\left(p_{\lambda} \otimes p_{\mu}\right)= & \sum_{g_{1}, g_{2} \in \mathfrak{S}_{p}} \chi_{V_{p}}\left(g_{1}, g_{2}\right) p_{\lambda}\left(g_{1}\right) p_{\mu}\left(g_{2}\right)= \\
& =\sum_{g_{1}, g_{2} \in \mathfrak{S}_{p}} \chi_{V_{p}}\left(g_{1} g_{2}^{-1}, \mathrm{id}\right) p_{\lambda}\left(g_{1}\right)(-1)^{g_{2}} p_{\mu}\left(g_{2}\right)=\chi_{V_{p}}\left(p_{\lambda} p_{\mu^{\prime}} \otimes 1\right) .
\end{aligned}
$$

The left-hand side is equal to the probability of sampling the pair $(\lambda, \mu)$; the right hand side vanishes unless $\lambda=\mu^{\prime}$ which concludes the proof.

\subsection{Proof of TheOrem 1.4.}

Proof of Theorem 1.4. In the light of the discussion from Section 3.9 the only remaining difficulty is to identify the distribution of the random Young diagram $\lambda$ which corresponds to the random irreducible component of the external power (1). By Schur-Weyl duality (Proposition 3.1) one can equivalently view $\lambda$ as a random Young diagram corresponding to a random irreducible component of the external power (1), viewed this time as a $Z \mathbb{C}\left[\mathfrak{S}_{p}\right]$-module in the specific way described in Section 3.8.

Equation (3.8) shows that in order to prove equality of the probability distributions of random Young diagrams associated to $Z \mathbb{C}[G]$-modules it is enough to prove equality of the corresponding characters. The latter equality of characters is provided by Corollary 3.3. In this way we proved the second part of Theorem 1.4 (the alternative formulation).

In order to finish the proof of the first part of Theorem 1.4 we notice that the branching rule implies that the distribution of the Young diagram which consists of boxes with enties $\leqslant p$ of a uniformly random standard Young tableau of fixed shape $\mu$ such that $|\mu| \geqslant p$ (in our setup we consider the special case $\mu=n^{m}$ ) coincides with the distribution of a Young diagram which corresponds to a random irreducible component of the restricted representation $V^{\mu} \downarrow_{\mathfrak{S}_{p}}^{\mathfrak{S}_{|\mu|}}$.

\subsection{Stanley CHARACTER FORMUla.}

REMARK 3.4. It is easy to use the ideas presented in the above proof to find a new elementary proof of Stanley's formula [14, Theorem 1] for the character of the symmetric group $\mathfrak{S}_{m n}$ which corresponds to the rectangular diagram $n^{m}$; for other proofs see also $[6,7]$. More specifically, one should calculate the character $\chi_{V_{p}}$ directly by calculating the trace $(10)$ in the standard basis of the tensor power $\left(\mathbb{C}^{m} \otimes \mathbb{C}^{n}\right)^{\otimes p}$ and apply Corollary 3.3 .

\section{Outlook}

We have to admit that in Section 1 we quoted only a part of the original question of Landsberg; in particular we have skipped the following more specific passage.

[...] I am most interested in the case where $p$ is near $\frac{m n}{2}$. Is there a slowly growing function $f(n)$ such that partitions with fewer than $f(n)$ steps contribute negligibly? If so, can the fastest growing such $f$ be determined?

Joseph M. Landsberg [9]

It is not clear if the ideas presented in this note are sufficient to tackle this more specific problem. For more on this topic see the work of Sevak Mkrtchyan [11]. 
Acknowledgements. We thank Paul Wedrich for pointing out the reference [8]. We thank Vadim Gorin for an interesting discussion. Figure 1 has been provided by Dan Romik.

\section{REFERENCES}

[1] Philippe Biane, Quantum random walk on the dual of SU(n), Probab. Theory Related Fields 89 (1991), no. 1, 117-129.

[2] _ Representations of unitary groups and free convolution, Publ. Res. Inst. Math. Sci. 31 (1995), no. 1, 63-79.

[3] _ Representations of symmetric groups and free probability, Adv. Math. 138 (1998), no. $1,126-181$

[4] Alexey Bufetov and Vadim Gorin, Representations of classical Lie groups and quantized free convolution, Geom. Funct. Anal. 25 (2015), no. 3, 763-814.

[5] Benoît Collins, Jonathan Novak, and Piotr Śniady, Semiclassical asymptotics of $\mathrm{GL}_{n}(\mathbb{C})$ tensor products and quantum random matrices, Preprint arXiv:1611.01892, 2016.

[6] Valentin Féray, Stanley's formula for characters of the symmetric group, Ann. Comb. 13 (2010), no. 4, 453-461.

[7] Valentin Féray and Piotr Śniady, Asymptotics of characters of symmetric groups related to Stanley character formula, Ann. of Math. (2) 173 (2011), no. 2, 887-906.

[8] Roger Howe, Perspectives on invariant theory: Schur duality, multiplicity-free actions and beyond, The Schur lectures (1992) (Tel Aviv), Israel Math. Conf. Proc., vol. 8, Bar-Ilan Univ., Ramat Gan, 1995, pp. 1-182.

[9] Joseph M. Landsberg, Distribution of Young diagrams, February 2012, Question asked on MathOverflow, https://mathoverflow.net/questions/88435/distribution-of-young-diagrams.

[10] Joseph M. Landsberg and Giorgio Ottaviani, New lower bounds for the border rank of matrix multiplication, Theory Comput. 11 (2015), 285-298.

[11] Sevak Mkrtchyan, On a question of J. M. Landsberg, In preparation, 2017.

[12] Boris Pittel and Dan Romik, Limit shapes for random square Young tableaux, Adv. in Appl. Math. 38 (2007), no. 2, 164-209.

[13] Piotr Śniady, Gaussian fluctuations of characters of symmetric groups and of Young diagrams, Probab. Theory Related Fields 136 (2006), no. 2, 263-297.

[14] Richard P. Stanley, Irreducible symmetric group characters of rectangular shape, Preprint arXiv:math/0109093, 2001

Greta Panova, UPenn Mathematics Department, 209 South 33rd St, Philadelphia, PA 19104, USA E-mail : panova@math.upenn.edu

Piotr Śniady, Institute of Mathematics, Polish Academy of Sciences, ul. Śniadeckich 8, 00956 Warszawa, Poland

E-mail : psniady@impan.pl 